\title{
PERFORMANCE OF A CONSTANT TORQUE PEDAL DEVICE
}

\author{
K. SHERWIN, PhD, BSc, MRAeS \\ Division of Engineering Design and Production, Department of Mechanical Engineering, \\ The University of Liverpool, P. O. Box 147, Liverpool L69 3BX.
}

\begin{abstract}
A constant-torque oscillatory pedal-crank device using vertical movement of the feet is described and its performance compared to a conventional rotational cycle. Using a generator to measure the power output the constant-torque device produced $33 \%$ less power and thus has no practical value as an alternative to the conventional pedal-crank system.
\end{abstract}

\section{INTRODUCTION}

Following the publication of a book on man powered flight (Sherwin, 1971), the author has received a steady stream of letters from inventors suggesting improved mechanisms for transmitting human power to replace the conventional pedal-crank and chain as used on most man powered aircraft.

The pedal-crank and chain are, of course, highly efficient, having been the subject of extensive development on bicycles so that the majority of mechanisms could be discounted as either unsuitable or impractical, especially as most were "paper designs" and had not been tried in practice. However, one particular device stood out from the rest, because not only was it suggested quite independently, by some dozen different people, but at least four of the people have built different versions of the basic device and spoken highly of its performance in practice. This device was the constant-torque pedal device, the name having been defined by Gibbons (1977) to describe the principle on which it worked.

Unfortunately no quantitative data was available to support the claims made of the constant-torque device and so the following investigation was carried out in order to provide comparative performance figures between the constant-torque and pedal-crank mechanisms.

\section{CONSTANT TORQUE DEVICE}

With a conventional pedal-crank the moment-arm of the pedal varies throughout each cycle with the horizontal distance of the pedal from the centre of the crank. This gives a cyclic variation in torque.

Assuming that the human providing the power can maintain a constant force throughout each down stroke of the legs and the moment-arm from the centre of the crank did not vary, then it would be possible to have a constant-torque through each part of the cycle. The advantage of such a system is illustrated by the torque- time curves in Figure 1, which indicates that ideally a constant-torque device could provide $\mathbf{5 0 \%}$ more power than a pedal-crank for the same effort.

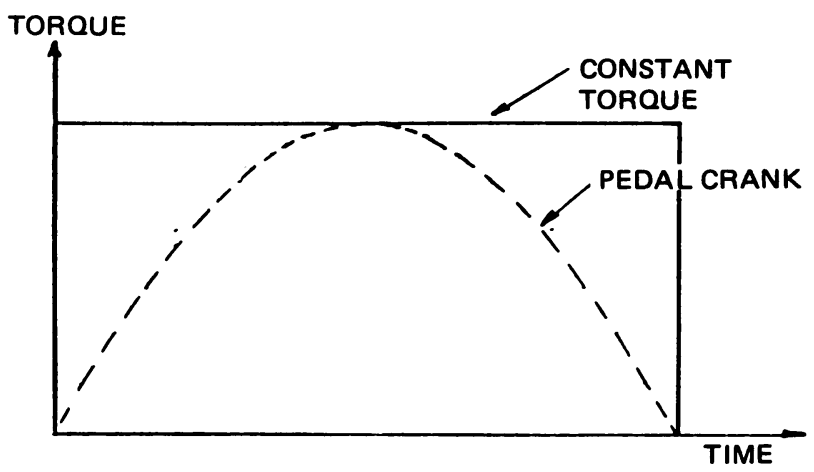

Figure 1. Torque-time curves for constant torque and pedal-crank systems.

Although such a huge increase was considered to be impossible to achieve in practice, the investigation was initiated because some evidence was available to indicate that the constant-torque device should give some improvement in power output. The improved torque characteristics of an ellipitical chain wheel produced a small improvement in power output compared to a circular chain wheel, Thompson, (1975). Furthermore, cyclists when wanting to apply a greater effort, when climbing up hills for example, tend to stand on the pedals which is more akin to a constant-torque application than to conventional cycling.

Several types of constant-torque devices have been designed, but the most straightforward to construct is that shown diagrammatically in Figure 2. The levers are sufficiently long for the movement at the ends to be nearly vertical, thereby providing a constant-torque throughout each down stroke. The drive is taken by either cable or chain, to a pair of ratchet mechanisms in order to provide a circular motion to the horizontally mounted output shaft. 


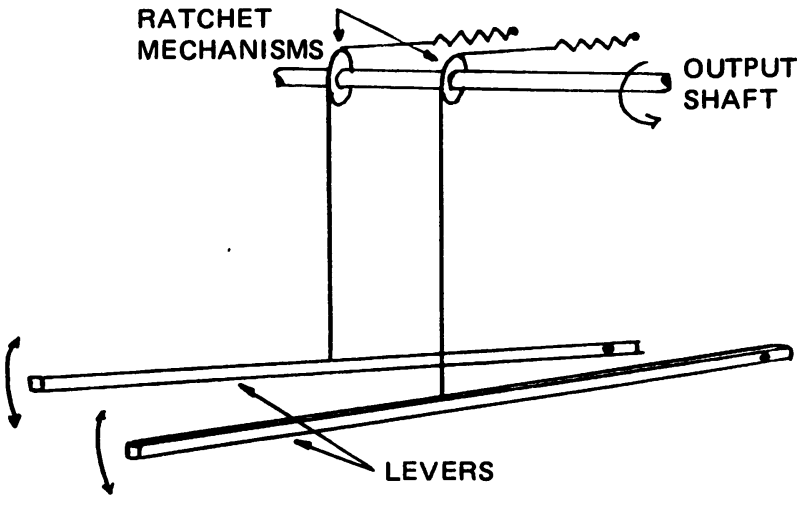

Figure 2. Diagram of a constant-torque device.

\section{FORM OF CONSTRUCTION}

In order to carry out the comparative performance tests, a constant-torque device of the type shown in Figure 2 was constructed. The actual rig used for this investigation is shown in Figure 3. The levers had a length of $1.5 \mathrm{~m}$ from the pivots to the pedals. This length was chosen on an arbitrary basis to provide a stroke of $34 \mathrm{~cm}$ vertically in order to compare directly with the maximum movement of the pedal crank. Steel tubing, $2.5 \mathrm{~cm}$ square, was used for the construction of the levers to provide the necessary rigidity within a lightweight structure.

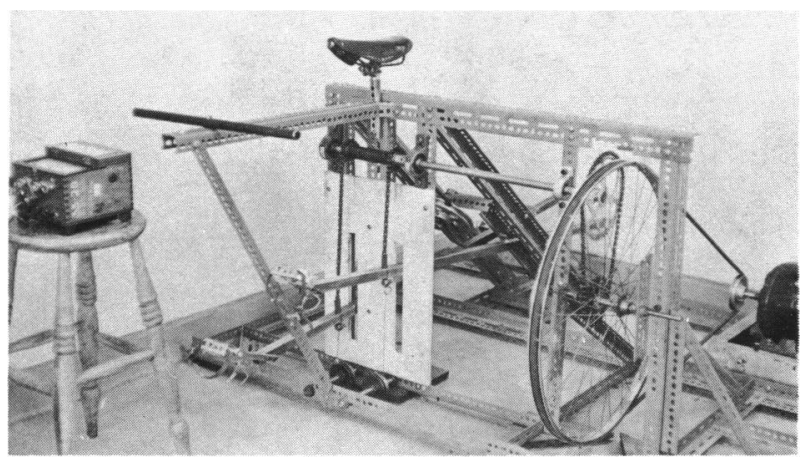

Figure 3. Construction of a constant-torque device.

Power from the levers was transmitted to the output shaft by means of two lengths of Renold's chain, each driving a cycle freewheel mechanism. The chains were tensioned by a cable joining the two together and passing over a horizontally mounted pulley.

To ensure that the comparison between the constanttorque device and the pedal-crank was as realistic as possible, bicycle pedals were attached to the end of the levers, complete with toe clips and straps. This meant that for lifting by the toe caps, further coupling of the levers was necessary and this was provided by a cable running under two pulleys below the levers.

The power was measured by coupling the device to a D.C. generator and reading the output in watts. To provide the step-up ratio necessary to drive the generator from the low rotational speed of the output shaft, a chain wheel was attached to the end of the output shaft. $A$ chain and fixed gear then drove a rear bicycle wheel and a V-belt driven from the periphery of the bicycle wheel drove a small pulley on the generator shaft.

\section{POWER OUTPUT}

In order to provide a direct comparison between the constant-torque device and a pedal-crank, a bicycle frame was incorporated within the rig in line with the constant-torque input levers. The crank was coupled to a fixed gear attached to the rear bicycle wheel driving the generator, thereby allowing the power input from a pedal-crank to be measured also.

Two students, who were both keen cyclists, were chosen to perform the comparative tests, which took place over a period of several weeks. The tests were performed by setting a constant power output and measuring the maximum duration during which each student could maintain power. It was appreciated that at each power output different subjects have different pedalling speeds, so that at each power level the field resistance of the D.C. generator was varied to allow each student to optimise his own performance..

The results of the tests for both the constant-torque device and the conventional pedal-crank for both students are given in Figure 4.



Figure 4. Power-duration curves for the constanttorque and pedal-crank systems. 


\section{DISCUSSION}

Although the investigation was carried out from an engineering basis, it is considered that the results presented in Figure 4 are realistic. The form of the results for the pedal-crank are consistent with the standard curves presented by Wilkie (1960). Also the higher power outputs for both the pedal-crank and constanttorque device were produced by the same student.

The results indicate that the constant-torque device produced a consistently lower power output than for the pedal-crank. At first this was thought to be due to the cycling experience of the students preventing them operating the constant-torque device in the most effective manner. To check this a non-cyclist was asked to produce 185 watts on both devices with a five hour rest period between. The durations achieved were $144 \mathrm{~s}$. for the pedal-crank compared to only $\mathbf{4 4} \mathrm{s}$. for the constanttorque device.

Several reasons can be suggested for the poor performance of the constant-torque device. In the first place there was some pawl lag in the free wheels, causing a rapid acceleration of the legs at the beginning of each down stroke, followed by a retardation as the torque started to be transmitted through each free wheel. This could have been overcome by more careful mechanical matching between the levers and the free wheels, but it is doubted whether this would have made a marked improvement to the power output of the constanttorque device.
What is of more significance is the wastage of energy in overcoming the inertia of the legs and of the long levers during each stroke. In this connection it seems to have certain similarities with rowing, where the power output cannot be increased for small durations due to the inertia of the moving parts. However, there the similarity ends because rowing produces the same power as a pedal-crank system for exercises of long duration, whereas the constant-torque device shows a reduction in power throughout the whole range.

\section{CONCLUSIONS}

On the basis of this particular investigation, a constanttorque device produces $33 \%$ less power than an equivalent pedal-crank system for the same human input. Therefore such a device has no practical value as an alternative to the conventional pedal-crank for human powered machines. ${ }^{*}$

\section{ACKNOWLEDGMENTS}

The author wishes to acknowledge the work of D. J. Bingham and D. J. Lawrie throughout the course of this investigation.

"Editors note: the "Kangaroo" tricycle of about 1885 used a similar pedal mechanism but no power measurements appear to have been made and the vehicle was too cumbersome to survive in town traffic.

\section{REFERENCES}

Sherwin, K. 1971. "Man powered flight". Model and Allied Publications, Hemel Hempstead.

Gibbons, T. S. 1977. Private communication.

Thompson, R. N. 1975. Project report. Department of Mechanical Engineering, Bradford University.

Wilkie, D. R. 1960. "Man as an aero engine". Journal of the Royal Aeronautical Society, August. 